\title{
Roles of Fascin mRNA expression in colorectal cancer: Meta-analysis and bioinformatics analysis
}

\author{
SHUAI SHI, HUA-CHUAN ZHENG and ZHI-GANG ZHANG \\ Department of Pathology, Cangzhou People's Hospital, Cangzhou, Hebei 061000, P.R. China
}

Received July 4, 2019; Accepted April 22, 2020

DOI: $10.3892 / \mathrm{mco} .2020 .2069$

\begin{abstract}
Fascin (encoded by FSCN1) is a globular actin cross-linking protein that is required for the formation of actin-based cell surface processes, which are critical for cell migration and cell-matrix adhesion. In the present study, a systematic meta-analysis and bioinformatics analysis was used to identify clinicopathological or prognostic parameters in patients with colorectal cancer. A total of 17 articles were included in the present study obtained from PubMed, Web of Science, Wanfang data, SinoMed and CNKI databases. Odd ratios (ORs) and the corresponding 95\% confidence intervals (CIs) were used to estimate the prognostic significance of Fascin expression in patients with colorectal cancer, and the association between Fascin expression and clinicopathological factors. There was a significant correlation between high Fascin expression and poor overall survival rates in patients with colorectal cancer $(\mathrm{OR}=0.48 ; 95 \% \mathrm{CI}$, $0.38-0.60 ; \mathrm{P}<0.000001)$. The meta-analysis showed that the expression of Fascin was significantly higher in colorectal cancer tissue compared with the normal mucosa $(\mathrm{OR}=0.13$; 95\% CI, 0.10-0.16; $\mathrm{P}<0.000001)$ and adenoma $(\mathrm{OR}=0.23$; 95\% CI, 0.15-0.34; P<0.000001). Fascin expression was also associated with depth of invasion $(\mathrm{OR}=0.31 ; 95 \% \mathrm{CI}$, 0.19-0.50; $\mathrm{P}<0.000001)$, lymph node metastasis $(\mathrm{OR}=3.07$; 95\% CI, 1.72-5.46; $\mathrm{P}=0.0001)$, Dukes stage $(\mathrm{OR}=0.14 ; 95 \%$ CI, 0.04-0.46; $\mathrm{P}=0.001)$, Tumor-Node-Metastasis stage $(\mathrm{OR}=0.38 ; 95 \% \mathrm{CI}, 0.21-0.71 ; \mathrm{P}=0.003)$ and dedifferentiation $(\mathrm{OR}=0.42 ; 95 \% \mathrm{CI}, 0.19-0.94 ; \mathrm{P}=0.04)$. According to the bioinformatics analyses, FSCN1 mRNA expression levels were higher in colorectal cancer and adenoma tissues compared with the normal tissues $(\mathrm{P}<0.05)$. According to TCGA, FSCN1 mRNA expression was associated with a less favorable prognosis in patients with colorectal cancer as an independent factor $(\mathrm{P}<0.05)$, and positively correlated with
\end{abstract}

Correspondence to: Professor Zhi-Gang Zhang, Department of Pathology, Cangzhou People's Hospital, 7 Qingchi Avenue, Cangzhou, Hebei 061000, P.R. China

E-mail: zhzhg001518@126.com

Key words: Fascin, colorectal cancer, meta-analysis, bioinformatics analysis, prognosis depth of invasion, microsatellite instability and low serum carcinoembryonic antigen levels in colorectal cancer. Taken together, the results of the present study suggested that Fascin expression is a potential marker of tumorigenesis, aggressiveness and poor prognosis in patients with colorectal cancer.

\section{Introduction}

Fascin is a cytoskeletal protein, is one of the important members of the Fascin family of proteins and is located on chromosome 7q22 (1). An N-terminal serine participates in actin binding, which is also the phosphorylation site of protein kinase $\mathrm{C}$. The phosphorylation of this site regulates the binding between Fascin and actin, and also increased the formation of pseudopodia on the surface of the cell membrane (1). Fascin is an actin-binding protein that can alter the cytoskeleton to form pseudopodia and microspores, increase the motility of epithelial cells, and reduce adhesion between cells and the extracellular matrix, thereby promoting cell migration (2), and also partakes in signal transduction (3). Fascin can catalyze the polymerization of actin and regulate the formation of microfilaments and filamentous pseudopodia on the cell surface, increasing the mobility of the epithelium and increasing cell surface projections, to increase cell migration, invasion and metastasis of tumor cells (4). Progression and invasion of colorectal cancer are closely associated with the increase in the motility of cancer cells, and the structural changes of the kinetic protein cytokine skeleton and the structure and function of the three-dimensional actuation microfilament are regulated and controlled by numerous kinetic protein binding proteins (5). Findings have shown that an increased expression of Fascin is closely associated with malignant biological behaviors, such as poor survival and metastasis in patients with colorectal cancer (6).

Fascin is involved in progression, survival and migration, and the expression of MMP-2 and collagen genes in human hepatic stellate cells, via a FAK-PI3K-AKT signaling pathway (7). PI3K may also regulate Skp2-mediated degradation of p27 through downstream signaling molecules (such as PKC, p70S6K and SGK, amongst others), and thus regulate the proliferation of liver cancer cells (8). Fascin is involved in STAT3 signaling in response to oncostatin $M$ and interleukin-6 in human breast cancer cells, STAT3 directly increases the expression of Fascin, and this underlies the migration of breast cancer cells (9). The STAT3-SKP2 molecular network 
controls the development of cervical cancer (10). Fascin serves an important role in laryngeal cancer cell transition from G1 to the $\mathrm{S}$ phase (11).

Fascin expression is upregulated in lung cancer, thyroid, ovarian and liver cancer (12-15), and is associated with a poor prognosis, Tumor-Node-Metastasis (TNM) staging and lymph node and distant metastases (16). Simultaneously, the expression of Fascin-1 in the outer layer of the tumor is higher compared with the inner tumor, suggesting that it may be associated with tumor invasion (17). The upregulation of Fascin in cells can result in increased in cell membrane process formation, dissociation of the cell from the extracellular matrix, and thus an increase in cell motility, which serves a key role in invasion and lymph node metastasis (18-20). Hayashi et al (21) detected the expression of Fascin-1 in the early stages of liver formation using immunohistochemistry. In particular, in the embryonic stage, the expression of Fascin-1 is significantly increased in liver buds and hepatocytes. These results suggest that the expression of Fascin-1 may be associated with migratory activity of hepatocytes during the early hepatogenesis (21). In the present study, a meta-analysis and bioinformatics analysis was performed to provide evidence of the association between Fascin expression and clinicopathological factors in patients with colorectal cancer.

\section{Materials and methods}

Literature search and selection criteria. Articles included in the present analysis were searched for in PubMed, Web of Science, Wanfang data, SinoMed and CNKI (June, 2019) using the following key words and modifiers: Fascin OR Fascin-1 OR FSCN1 AND colorectal OR colon OR rectum OR rectal AND cancer OR carcinoma OR tumor OR adenocarcinoma. Inclusion criteria for studies were: i) Studies using immunohistochemistry to detect the expression of Fascin or Fascin-1; ii) articles which included an association between Fascin expression and prognosis in colorectal cancer; and iii) articles assessing the association between Fascin expression and clinicopathological parameters, such as depth of invasion, lymph node metastasis and TNM stages, amongst others. The exclusion criteria were: i) Abstracts, case reports, reviews and meeting notes; ii) studies with a small sample size $(\mathrm{n}<30)$; iii) repeat publications or repeat data; and iv) and animal-based studies.

Data extraction and quality assessment. The information regarding all eligible publications was extracted by two reviewers, and the authors, year of publication, nationality of the patients, antibody companies, number of cases and controls, risks for cancer and follow-up outcomes are presented in Table I. Any disagreements regarding any of these data were resolved by discussion. The quality of the studies was independently assessed by two reviewers according to Newcastle Ottawa Oncomine Scale (NOS; ohri.ca/programs/clinical_epidemiology/oxford.htm). The methods were assessed based on consistency of sample selection, comparability and ascertainment of outcomes.

Bioinformatics analysis. FSCN1 gene expression levels were analyzed using Oncomine (oncomine.org), the largest chip-based oncogene database and integrated data mining platform. Multiple analysis (fold change) and the expression ratio of FSCN1 in the range of 0.5-2.0, and there was no significant differential expression of the gene. Genes where the T statistic (T-test) exceeded a specific value was considered an abnormality. Whether the comparison was statistically significant was determined by calculating the confidence of the difference. The differences in FSCN1 mRNA expression levels were compared between colorectal tissue (including the colon and the rectum), normal tissue, adenoma and colorectal cancer. All data were log-transformed, median centered per array, and the standard deviation was normalized to a single value for each array. The expression data were obtained (RNA-seqV2) and clinicopathological data of colorectal cancer from The Cancer Genome Atlas (TCGA) database (cancer.gov) were analyzed using TCGA-assembler in R software. The raw data were integrated, $F S C N 1$ expression in colorectal cancer was analyzed and compared with the clinicopathological and prognostic data of patients with colorectal cancer.

Statistical analysis. Revman version 5.3 (cochrane.es) was used for data analysis. Odds ratios (ORs) and 95\% confidence intervals (CIs) were used to estimate the expression of Fascin based on the clinicopathological parameters of patients with colorectal cancer. Initially, the heterogeneity of the original documents were assessed. Statistical significance of the pooled ORs were determined using $\mathrm{Z}$ tests. If there was no significance in the heterogeneity, a fixed effect model (Mantel-Haenszel method) was used, otherwise, a random effect model (Der Simonian and Laird method) was used. The effect of heterogeneity was quantified using an $\mathrm{I}^{2}$ test. Using the following cut-off values; 25,50 and $75 \%$, heterogeneity was subdivided into low, medium and high degrees, respectively. Publication bias was evaluated using funnel plot and quantified using Begg's test and Egger's test to assess funnel plot asymmetry. A funnel plot was used to evaluate publication bias. COX risk regression models were used for univariate and multivariate analysis. Meta-analyses were performed using Revman 5.3 and data obtained from TCGA was analyzed using SPSS version 17.0, and compared using a Student's t-test. Two-sided $\mathrm{P}<0.05$ was considered to indicate a statistically significant difference.

\section{Results}

Study selection and characteristics. As shown in Fig. 1, a total of 17 articles were found which assessed the relationship between Fascin expression and clinical pathology or prognosis of patients with colorectal cancer. Only 14 articles included analysis of normal colorectal tissue (22-35), and 6 included analysis of colorectal adenoma $(24,27,29,32-34)$. These 15 articles were used in the meta-analysis for comparison between Fascin expression and clinicopathological features of colorectal cancer. Additionally, there were 4 articles that discussed the prognostic significance of Fascin expression and its relationship clinicopathological or prognostic indicators of colorectal cancer $(23,29,30,36)$. All these studies evaluated the expression of Fascin and the risk of colorectal cancer using immunohistochemistry. The detailed characteristics of these articles are presented in Table I. 
Table I. Primary characteristics of the eligible studies.

\begin{tabular}{|c|c|c|c|c|c|c|c|c|c|}
\hline Author, year & Country & Ethnicity & Antibody supplier & Cases & Control & Risk to cancer & Outcome & Quality & (Refs.) \\
\hline Zhao, 2014 & China & China & & 126 & 126 & Increased & - & 7 & (31) \\
\hline Yi, 2013 & China & China & Sunbio & 60 & 60 & Increased & - & 8 & (34) \\
\hline Xue, 2010 & China & USA & Dako & 28 & 5 & Increased & - & 8 & (28) \\
\hline Song, 2010 & China & China & $\mathrm{Mxb}$ & 40 & 20 & Increased & - & 8 & (32) \\
\hline Chan, 2010 & Australia & USA & Dako & 446 & 433 & Increased & Negative & 9 & (29) \\
\hline Yao, 2014 & China & China & $\mathrm{Mxb}$ & 40 & 20 & Increased & Negative & 8 & (30) \\
\hline Yang, 2006 & China & USA & Dako & 41 & 20 & Increased & - & 8 & (33) \\
\hline Seung, 2012 & Korea & USA & Dako & 74 & 52 & Increased & - & 8 & (35) \\
\hline Kong, 2016 & China & USA & Clplor & 87 & 28 & Increased & Negative & 8 & (23) \\
\hline $\mathrm{Li}, 2015$ & China & China & $\mathrm{Mxb}$ & 86 & 40 & Increased & - & 8 & (24) \\
\hline Liu, 2007 & China & China & Mxb & 80 & 40 & Increased & - & 8 & $(25)$ \\
\hline Ding, 2008 & China & China & Chemicon & 100 & 100 & Increased & - & 8 & (26) \\
\hline $\mathrm{Li}, 2010$ & China & China & Mxb & 60 & 30 & Increased & - & 8 & $(27)$ \\
\hline Jung, 2011 & Korea & USA & Thermo Fisher Scientific, Inc. & 186 & 24 & Increased & Negative & 8 & (36) \\
\hline Pang, 2011 & China & USA & Neomarker & 60 & 20 & Increased & - & 8 & (22) \\
\hline
\end{tabular}

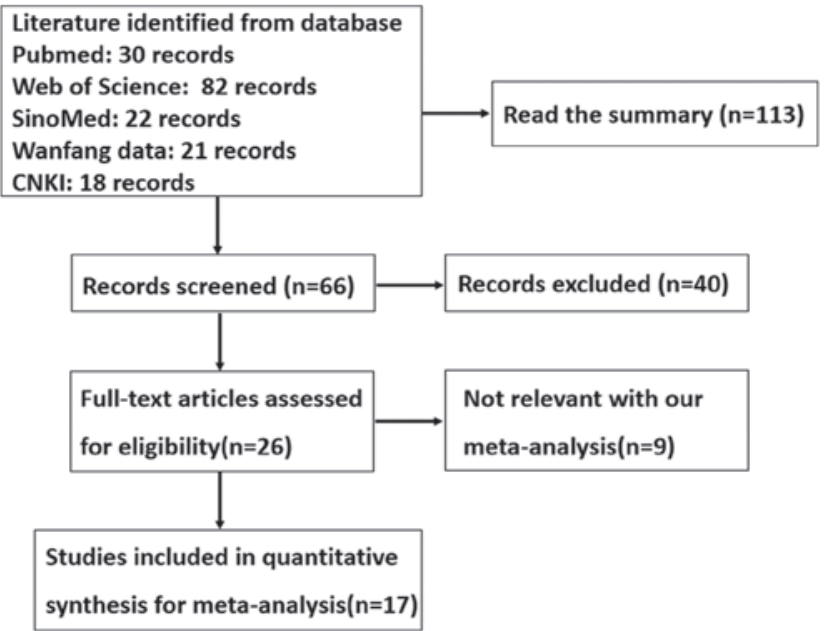

Figure 1. Flow diagram of article selection.

Forest plot of OR for the association between Fascin expression and clinicopathological parameters of colorectal cancer. The relationship between Fascin expression and tumor susceptibility in colorectal normal mucosa tissues were assessed in 14 studies, with 1,484 cancer patients and 1,004 controls collectively. The expression of Fascin was upregulated in colorectal cancer compared with normal mucosa (Fig. 2A; OR=7.78, 95\% $\mathrm{CI}=6.14-9.87, \mathrm{P}<0.00001)$. Additionally, the cancer risk of Fascin-positive adenoma was also assessed, and the same trend were observed as in colorectal cancer using 329 cancer cases and 236 adenoma cases (Fig. 2B; OR=0.23, 0.15-0.34, $\mathrm{P}<0.00001$ ). The meta-analysis showed that Fascin expression was associated with depth of invasion $(\mathrm{OR}=0.31,95 \% \mathrm{CI}=0.19-0.50, \mathrm{P}<0.00001)$, lymph node metastasis $(\mathrm{OR}=3.07,95 \% \mathrm{CI}=1.72-5.46, \mathrm{P}=0.0001)$, Dukes stage $(\mathrm{OR}=0.14,95 \% \mathrm{CI}=0.04-0.46, \mathrm{P}=0.0001)$, dedifferentiation $(\mathrm{OR}=0.42,95 \% \mathrm{CI}=0.19-0.94, \mathrm{P}=0.04)$ and TNM stage
$(\mathrm{OR}=0.38,95 \% \mathrm{CI}=0.21-0.71, \mathrm{P}=0.003)$ (Fig. 2C-H). However, Fascin was not associated with distant metastasis $(\mathrm{OR}=2.26,95 \%$ $\mathrm{CI}=0.78-6.51, \mathrm{P}=0.13)$. The survival data are presented in Fig. 2I, and based on the 4 datasets, it was shown that Fascin expression was associated with a less favorable prognosis in patients with colorectal cancer (hazard ratio $=0.48,95 \% \mathrm{CI}=0.38-0.60$, $\mathrm{P}<0.00001)$.

Publication bias. Publication bias was qualitatively determined using funnel plots (Fig. 3). An individual study was removed from the pooled analysis, and then sensitivity analysis was used to assess the impact of that individual study on the aggregated results. According to Egger's test, the present meta-analysis had no apparent publication bias.

Relationship between Fascin expression and bioinformatics analysis in patients with colorectal cancer. According to Hong's datasets, FSCN1 mRNA expression was upregulated in colon carcinoma tissues compared with the normal tissues (Fig. 4A, P<0.05). In Kaiser and TCGA dataset, FSCN1 mRNA expression was also upregulated in colon mucinous adenocarcinoma tissues compared with colon tissues (Fig. 4B and C, $\mathrm{P}<0.05)$. Notterman's and Ki's data showed increased FSCN1 mRNA expression levels in colon adenocarcinoma tissues compared with the normal colon tissue (Fig. 4D and E, P<0.05). According to Gaedcke's data, the expression of FSCN1 mRNA in rectal adenocarcinoma was higher compared with normal tissue (Fig. 4F, P<0.05). Skrzypczak's data showed that the expression of FSCN1 mRNA was higher in colon adenoma and colon carcinoma compared with normal tissue (Fig. 4G-J, $\mathrm{P}<0.05$ ), and also showed that FSCN1 mRNA expression was upregulated in colorectal carcinoma compared with colorectal tissue (Fig. 4K, $\mathrm{P}<0.05$ ).

According to the data obtained from TCGA, univariate analysis showed a positive correlation between FSCN1 mRNA 


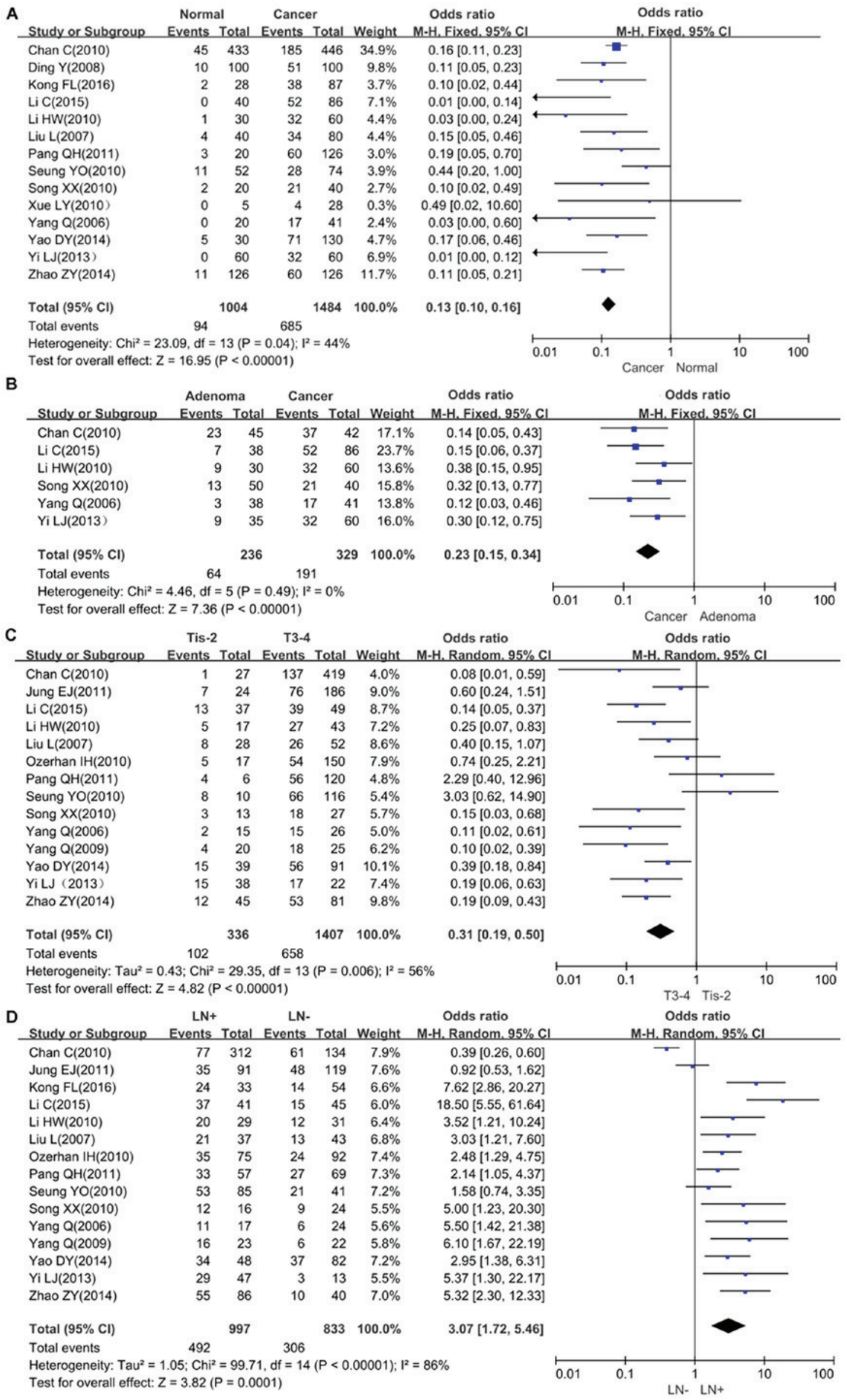

Figure 2. Forest plot of the relationship between Fascin expression and clinicopathological parameters of patients with colorectal cancer. Plots of the association between (A) cancer and normal mucosa, (B) cancer and adenoma, (C) depth of invasion ( $\mathrm{T}_{\mathrm{is}-2}$ and $\left.\mathrm{T}_{3-4}\right),(\mathrm{D})$ lymph node metastasis [LN and (LN- and $\left.\left.\mathrm{LN}+\right)\right]$. 


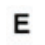

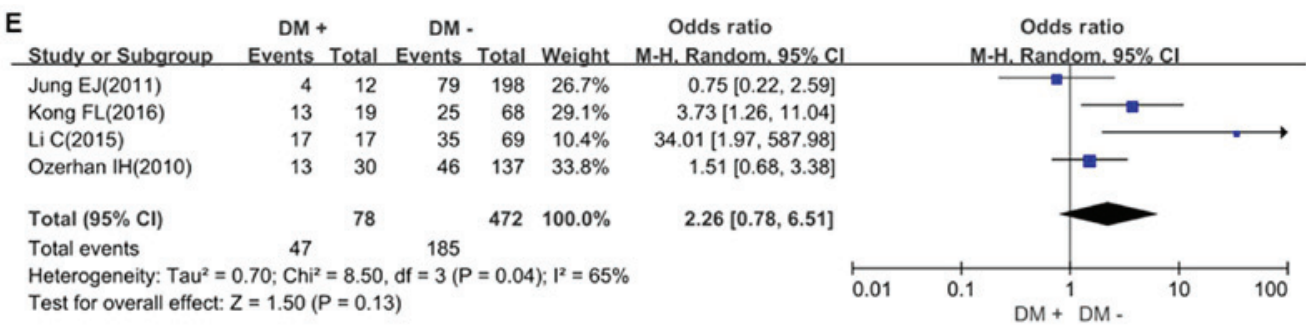

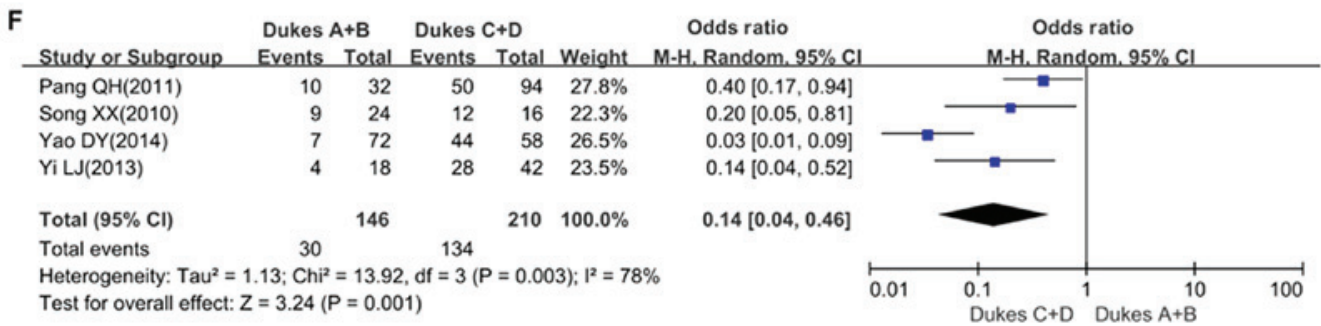

G

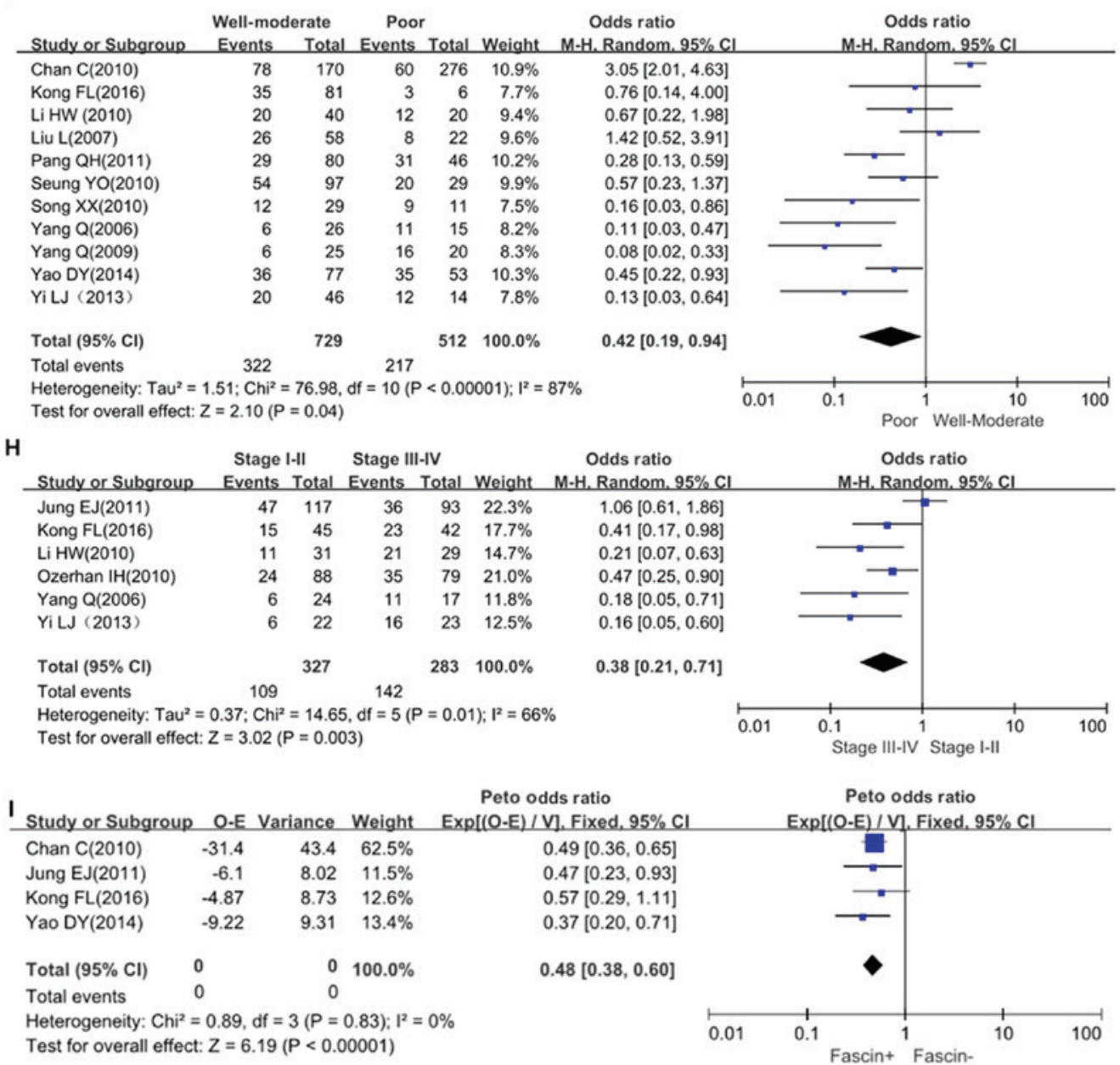

Figure 2. Continued. Forest plot of the relationship between Fascin expression and clinicopathological parameters of patients with colorectal cancer. Plots of the association between (E) DM (DM-vs. DM+), (F) Dukes stage (A+B vs. C+D), (G) dedifferentiation (dedifferentiation- vs. dedifferentiation+), (H) TNM stage (I-II and III-IV) and (I) survival (Fascin+ vs. Fascin-). CI, confidence interval; TNM, Tumor-Node-Metastasis; DM, distant metastasis.

expression and overall prognosis in patients with colorectal cancer (Fig. 4L, P<0.05). Multivariate analysis was performed using a Cox risk scale model, which showed that distant metastasis was an independent prognostic factor for colorectal cancer (Table II, $\mathrm{P}<0.05$ ). Fascin expression was positively correlated with depth of invasion and microsatellite instability (MSI) of colorectal cancer (Fig. $4 \mathrm{M}$ and $\mathrm{N}, \mathrm{P}<0.05$ ). These data also showed that FSCN1 mRNA expression levels were higher in the colon compared with the rectum (Fig. 4O, P<0.05), and that upregulated FSCN1 mRNA expression levels were negatively correlated with serum carcinoembryonic antigen (CEA) levels in all patients with cancer (Fig. 4P, $\mathrm{P}<0.05$ ). 
Funnel plot with pseudo $95 \%$ confidence limits

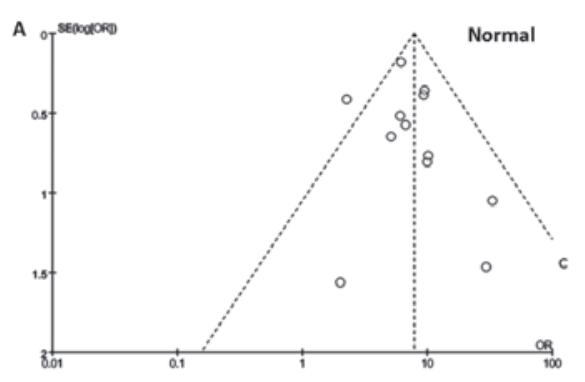

Funnel plot with pseudo $95 \%$ confidence limits

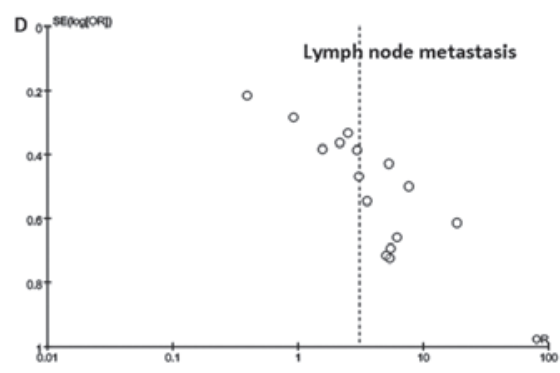

Funnel plot with pseudo $95 \%$ confidence limits

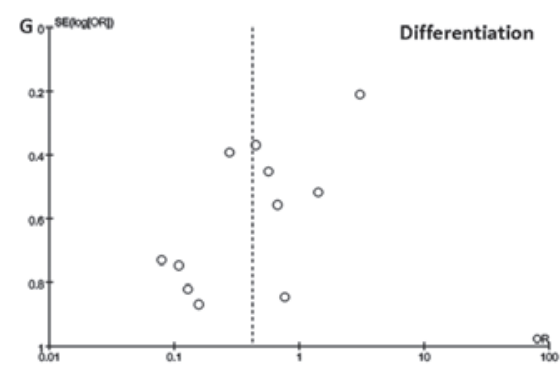

Funnel plot with pseudo $95 \%$ confidence limits

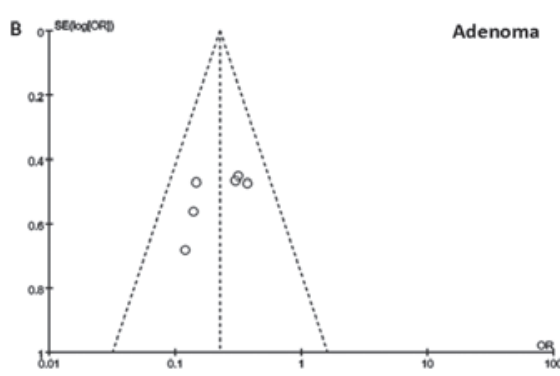

Funnel plot with pseudo $95 \%$ confidence limits

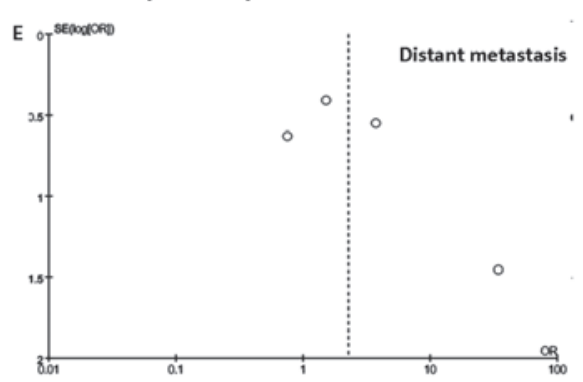

Funnel plot with pseudo $95 \%$ confidence limits

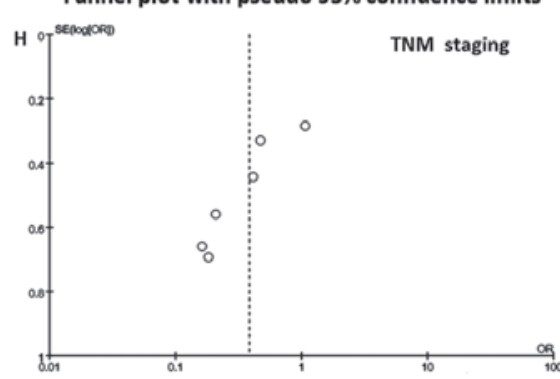

Funnel plot with pseudo $95 \%$ confidence limits

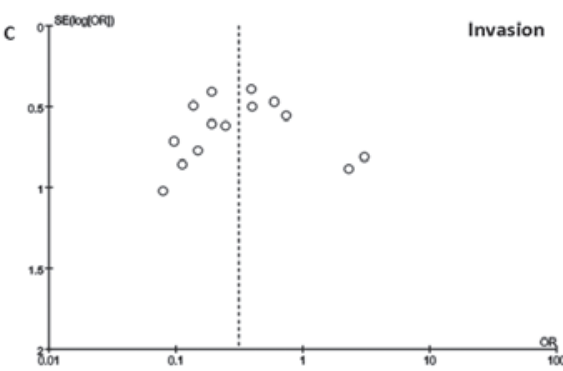

Funnel plot with pseudo $95 \%$ confidence limits

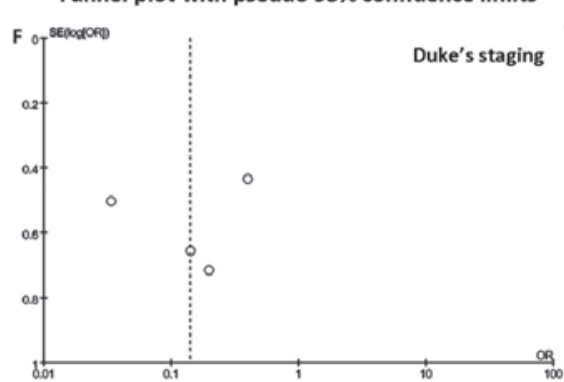

Funnel plot with pseudo $95 \%$ confidence limits

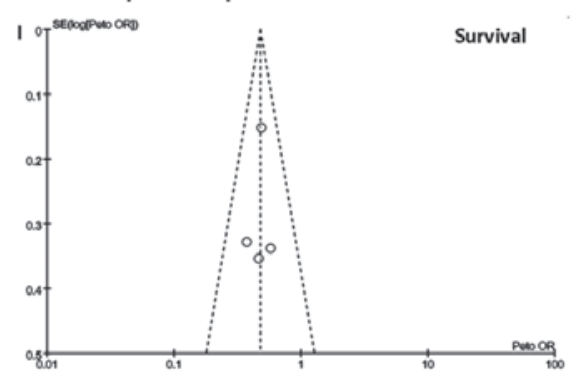

Figure 3. Funnel plot for testing publication bias between Fascin expression and colorectal carcinogenesis. Publication bias was analyzed based on risk degrees of Fascin expression in (A) colorectal mucosa and (B) adenoma for colorectal carcinogenesis. Additionally, publication bias was also tested between Fascin expression and clinicopathological features of colorectal cancer, (C) including depth of invasion, (D) lymph node metastasis, (E) distant metastasis, (F) Dukes stage, $(\mathrm{G})$ differentiation, (H) TNM stage and (I) survival. TNM, Tumor-Node-Metastasis.

\section{Discussion}

The Fascin family of proteins consists of 3 members; Fascin-1-3 (37-39). Fascin-1 is widely expressed in smooth muscle tissues, vascular endothelium, fibroblasts and in neural crest cells $(40,41)$. Fascin-2 expression is confined to retinal photoreceptor cells, and Fascin-3 is only expressed in the testis $(38,39)$. Fascin has been studied in different types of tumors, such as breast cancer, colon cancer, brain cancer, esophageal cancer, stomach cancer, lung cancer, urinary bladder cancer and hematological malignancies (42-48). In normal epithelia of the bile duct, breast, colon, ovary, pancreas and stomach, the expression of Fascin is usually completely negative (49). Increased expression of Fascin is associated with a less favorable prognosis in patients with lung, gastric, esophageal or breast cancer $(45,46,50,51)$. To demonstrate the association between Fascin expression and its clinicopathological features, a meta-analysis of 17 articles was performed which were selected based on the NOS scores.

Fascin expression is upregulated in colorectal cancer, but is not expressed in normal colorectal tissues. The studies showed that the expression of Fascin was associated with an invasive phenotype and a poor prognosis in patients with colorectal cancer. Thus, it is hypothesized that Fascin protein is universally expressed in epithelial tumors (35). Fascin expression was negatively associated with distant metastases. In cell culture, in cells treated with recombinant Fascin, the migratory and invasive capacities of colorectal cancer cells are increased (40). Similar findings have been obtained in other types of epithelial cells, suggesting that Fascin may facilitate a more aggressive tumor phenotype $(18,51)$. In the present study, Fascin protein expression was found to be positively associated with depth of invasion, lymph node metastasis, Dukes stage, dedifferentiation and TNM stage.

The mechanism by which Fascin is upregulated during tumorigenesis, invasion and metastasis may be associated with multiple signaling pathways. Previous reports suggest that amplification or overexpression of c-erbB-2/HER-2 may affect the upregulation of Fascin expression (52). A study found that Wnt signaling may also have an effect on Fascin activity, suggesting that anomalies in the Wnt signaling pathway may result in upregulation of Fascin expression in tumor cells (53). Exogenous TGF- $\beta$ and EGF induce the expression of Fascin, and this induction is dependent on the activation of ERK $(54,55)$, a downstream molecule of the Ras signaling pathway. Inhibition of phosphorylation of ERK 


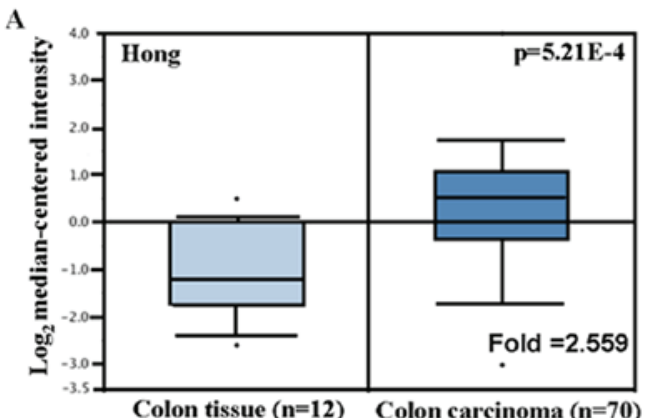

C

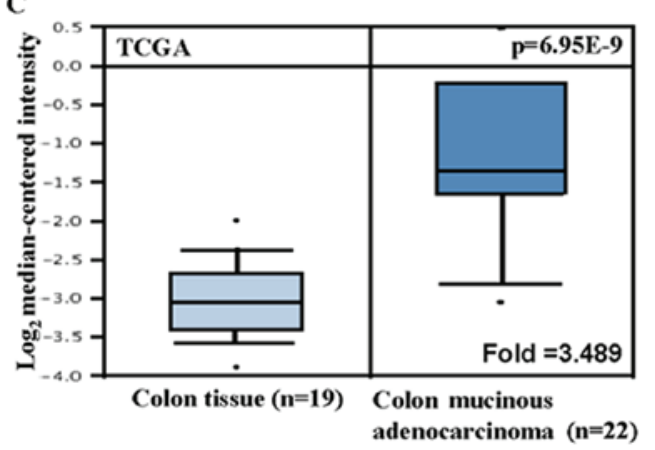

E

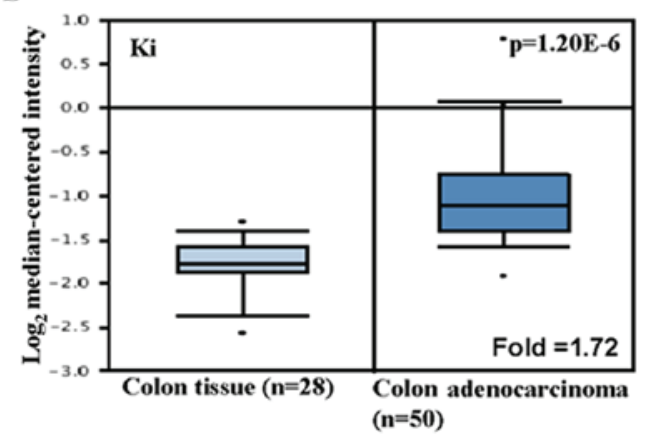

G
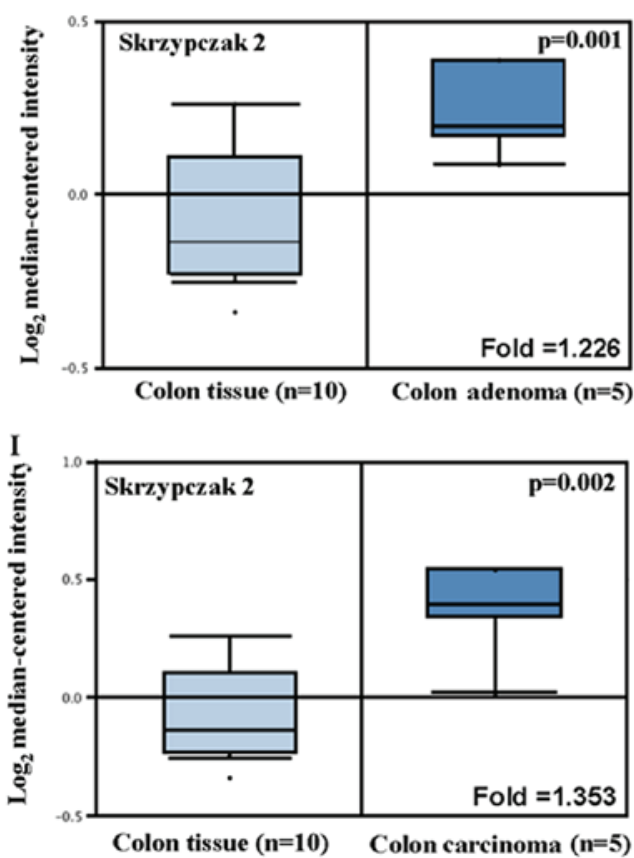

B

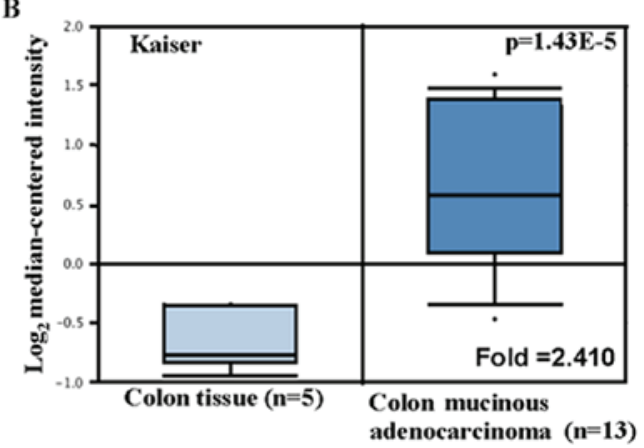

D

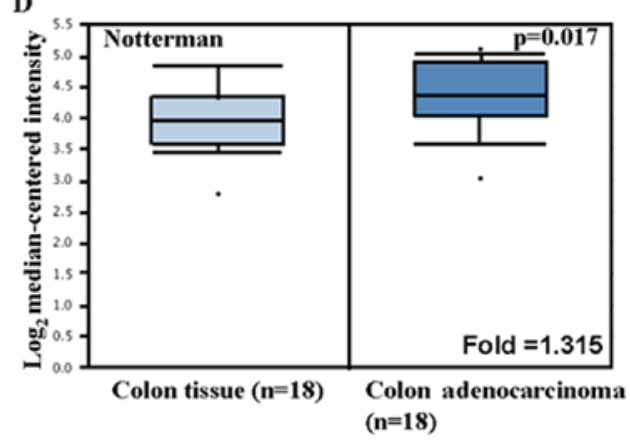

F

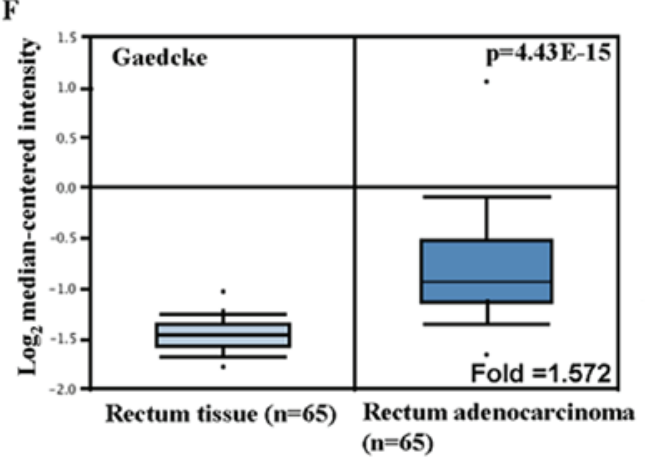

H

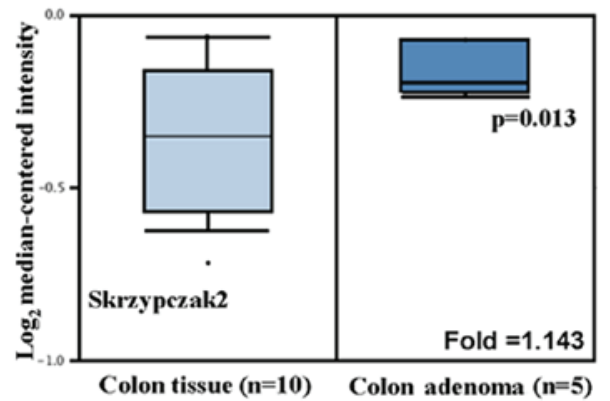

J

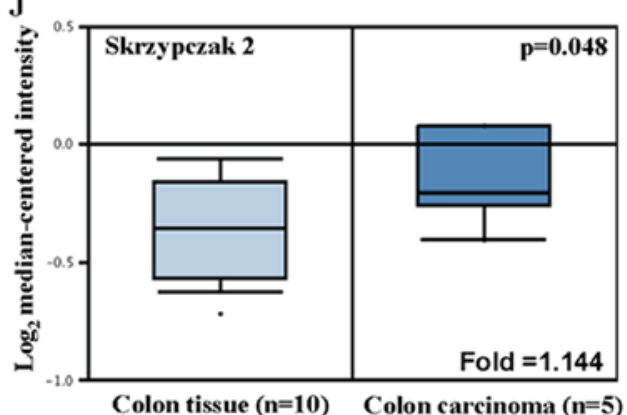

Figure 4. FSCN1 mRNA expression in colorectal carcinogenesis. (A) Expression of Fascin was significantly upregulated in colon carcinoma compared with the normal colon mucosa. (B) TCGA and (C) Kaiser databases showed that FSCN1 was upregulated in colon mucinous adenocarcinoma compared with the normal colon mucosa. (D and E) FSCN1 expression was higher in colon adenocarcinoma compared with normal colon mucosa and (F) adenocarcinoma. $(\mathrm{G}$ and $\mathrm{H}$ ) Based on the Skrzypczak2 database, FSCN1 upregulation in colon adenoma was higher compared with normal colon mucosa. (I and J) Expression of Fascin was significantly upregulated in colon carcinoma compared with the normal colon mucosa based on the Skrzypczak2 database. 

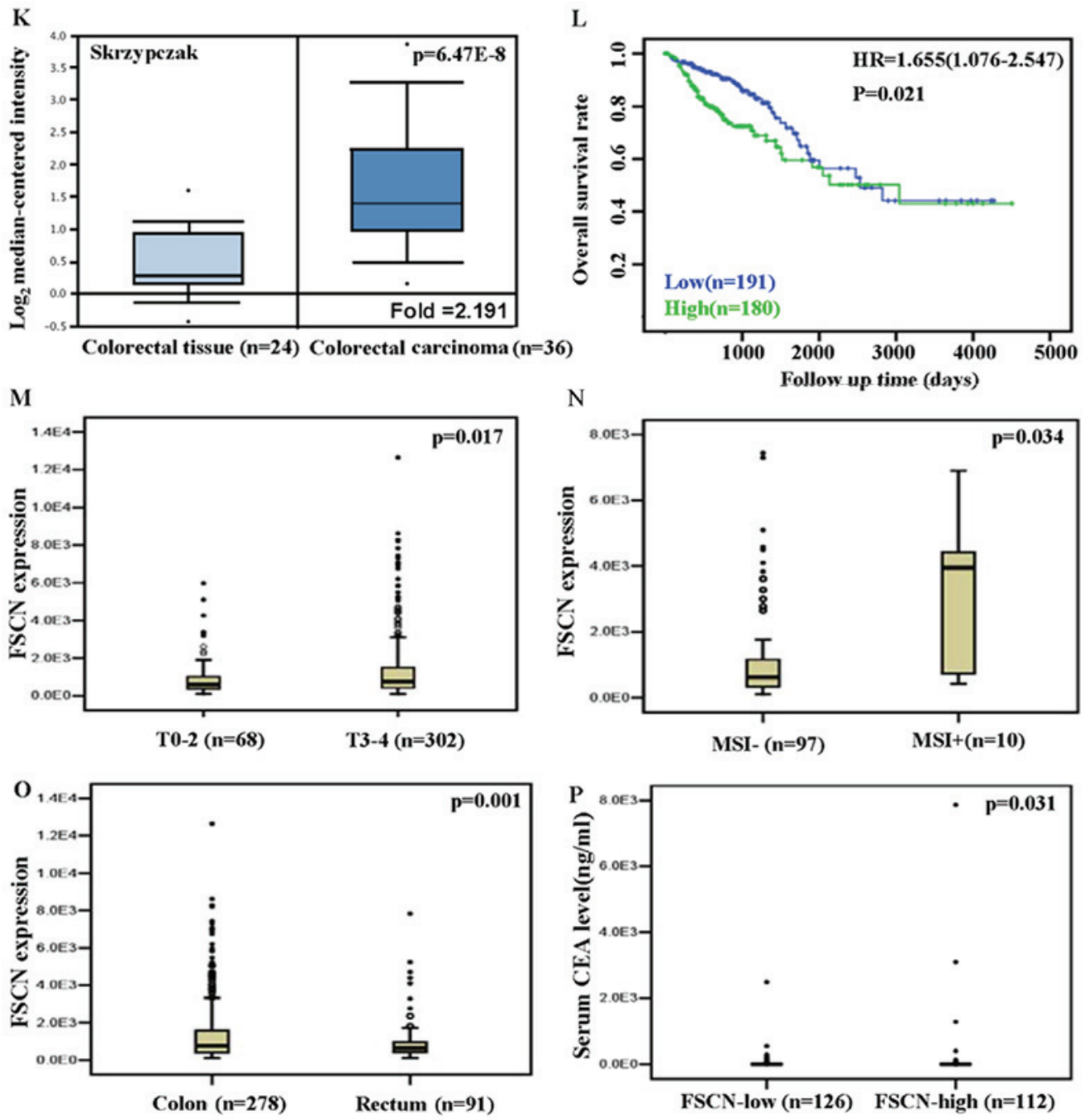

Figure 4. Continued. (K) Expression of Fascin was significantly upregulated in colon carcinoma compared with the normal colon mucosa based on the Skrzypczak database. (L) Univariate analysis showed a positive relationship between FSCN1 mRNA expression and an overall improved prognosis in patients with colorectal cancer. (M and N) Fascin expression was positively correlated with depth of invasion and MSI in colorectal cancer. (O) FSCN1 mRNA expression was higher in the colon cancer compared with rectal. (P) Increased FSCN1 mRNA expression was negatively correlated with serum CEA levels in all patients with cancer. HR, hazard ratio; FSCN, Fascin; MSI, microsatellite instability; CEA, carcinoembryonic antigen; TCGA, The Cancer Genome Atlas.

results in a decrease in the expression levels of Fascin (54), suggesting that activation of the downstream molecules of the ERK-Ras pathway results in increased Fascin expression in tumor cells. Fu et al (54) found a significant upregulation of Fascin expression in experimental autoimmune neuritis sciatic nerves which was associated with disease severity. The interaction between Fascin and PKC may be regulated by cannabinoid signaling, which controlled neuroblast migration in vivo. It was also shown that Fascin upregulation marked a shift to the migratory neuroblast stage and was a key regulator of neuroblast activity (55). Fascin can stabilize actin bundles in the invasive pseudopod structure, which may promote the metastatic potential of tumor cells $(56,57)$. Additionally, Fascin promotes epithelial-mesenchymal transition through the formation of pseudopodium, and formation of a mesothelial cell layer (58). Previous studies showed that LMP1 mediated the upregulation of Fascin, and this was dependent on NF- $\kappa \mathrm{B}$, where both NF- $\mathrm{KB}$ and Fascin contributed to an invasion and migration in LMP1-expressing lymphocytes (59). Fascin expression is significantly associated with Tiam1 expression. Tiam 1 activates Rac, and activated Rac affects the movement of cytoskeleton by inducing actin aggregation at the plasma membrane, resulting in formation of filopodia, and thus inducing invasion and metastasis of tumor cells (26).

Zhang et al (60) showed there was a positive correlation between Twist and Fascin mRNA expression levels in colorectal cancer, suggesting that they both served a synergistic role in the development and progression of colorectal cancer. In the present study, it was shown that there was a positive correlation between FSCN1 mRNA expression levels and overall prognosis in patients with colorectal cancer, and distant metastasis was an independent prognostic factor for colorectal cancer. However, Fascin protein expression levels were not associated with distant metastasis, and this may be due to the sample size. Simultaneously, Fascin expression was positively correlated with depth of invasion. In the univariate analysis, there may be false or indirect associations between independent and dependent variables, which are adjusted for and thus may disappear in the multivariate analysis. The purpose of multivariate analysis is to determine the influencing 
Table II. Multivariate analysis of hazardous factors in the prognosis of patients with colorectal cancer.

\begin{tabular}{llc}
\hline $\begin{array}{l}\text { Clinicopathological } \\
\text { features }\end{array}$ & \multicolumn{1}{c}{$\begin{array}{c}\text { Hazard } \\
\text { ratio }(95 \% \mathrm{CI})\end{array}$} & P-value \\
\hline T-stage, Tis $_{-2} / \mathrm{T}_{3-4}$ & $1.265(0.477-3.354)$ & 0.636 \\
Lymph node status, -/+ & $0.774(0.176-3.391)$ & 0.734 \\
Distant metastasis, -/+ & $2.247(1.212-4.165)$ & 0.010 \\
TNM staging, I-II/III-IV & $2.674(0.541-13.212)$ & 0.227 \\
FSCN mRNA expression & $1.430(0.877-2.330)$ & 0.151 \\
\hline
\end{tabular}

CI, confidence interval.

factors with independent effects, and to estimate the effect size by controlling the influence of other confounding factors.

The present study has some limitations. First, potential publication bias stems from the fact that published results were predominantly positive. Second, the patients included in the studies were only from Asia and America. Different levels of medical development in different areas may influence the results. Third, extraction of survival data, such as incomplete extraction, may affect results. Fourth, small sample sizes may influence associations in some articles. Finally, the difference in the expression of Fascin between normal tissues and adenoma was not assessed.

In conclusion, Fascin expression is upregulated in colorectal cancer and adenoma. Fascin expression was positively associated with depth of invasion, lymph node metastasis, Dukes stage, TNM staging, dedifferentiation and a less favorable prognosis in patients with colorectal cancer. FSCN1 mRNA expression was positively associated with depth of invasion, MSI, low serum CEA levels in colorectal cancer and with a less favorable prognosis in patients with colorectal cancer as an independent factor.

\section{Acknowledgements}

Not appliable.

\section{Funding}

Not funding was received.

\section{Availability data and materials}

The datasets used and/or analyzed during the present study are available from the corresponding author on reasonable request.

\section{Authors' contributions}

SS and ZGZ performed the meta-analysis, and wrote the manuscript. HCZ analyzed the data from TCGA. All authors approved the final manuscript.

\section{Ethics approval and consent to participate}

Not applicable.

\section{Patient consent for publication}

Not applicable.

\section{Competing interests}

The authors declare that they have no competing interests.

\section{References}

1. Li LJ, Xu L and Xia D: Progress of Fascin in colorectal cancer. J Mil Surg Southwest China 14: 753-756, 2012.

2. Zhou C and Li TY: Research progress of the relationship between fascin protein and tumor. J Nanchang Univ (Medical Sciences) 53: 94-96, 2013.

3. Hashimoto Y, Kim DJ and Adams JC: The roles of fascins in health and disease. J Pathol 224: 289-300, 2011.

4. SwierczynskiSL,Maitra A, Abraham SC,Iacobuzio-DonahueCA Ashfaq R, Cameron JL, Schulick RD, Yeo CJ, Rahman A, Hinkle DA, et al: Analysis of novel tumor markers in pancreatic and biliary carcinomas using tissue microarrays. Hum Pathol 35: 357-366, 2004.

5. Buda A and Pignatelli M: Cytoskeletal network in colon cancer: From genes to clinical application. Int J Biochem Cell Biol 36: 759-765, 2004.

6. Tsai WC, Chao YC, Sheu LF, Chang JL, Nieh S and Jin JS: Overexpression of fascin-1 in advanced colorectal adenocarcinoma: Tissue microarray analysis of immunostaining scores with clinicopathological parameters. Dis Markers 23: 153-160, 2007.

7. Uyama N, Iimuro Y, Kawada N, Reynaert H, Suzumura K, Hirano T, Kuroda N and Fujimoto J: Fascin, a novel marker of human hepatic stellate cells, may regulate their proliferation, migration, and collagen gene expression through the FAK-PI3K pathway. Lab Invest 92: 57-71, 2012.

8. Gungor-Ordueri NE, Celik-Ozenci C and Cheng CY: Fascin 1 is an actin filament-bundling protein that regulates ectoplasmic specialization dynamics in the rat testis. Am J Physiol Endocrinol Metab 307: E738-E753, 2014

9. Snyder M, Huang XY and Zhang JJ: Signal transducers and activators of transcription 3 (STAT3) directly regulates cytokine-induced fascin expression and is required for breast cancer cell migration. J Biol Chem 286: 38886-38893, 2011.

10. Huang H, Zhao W and Yang D: Stat3 induces oncogenic Skp2 expression in human cervical carcinoma cells. Biochem Biophys Res Commun 418: 186-190, 2012.

11. Wang J, Li X, Liu X and Pi L: The regulation of stat 3 signal transduction pathway to $\mathrm{G} 1$ to $\mathrm{S}$ phase of laryngocarcinoma cell. Lin Chung Er Bi Yan Hou Tou Jing Wai Ke Za Zhi 22: 699-703, 2008 (In Chinese)

12. Zhang N, Shen Y and Hu G: Expression of fascin protein in human lung cancer and its clinical significance. J Nanjing Med Univ (Natural Sciences) 30: 309-311, 2010.

13. Chen G, Zhang FR, Ren J, Tao LH, Shen ZY, Lv Z, Yu SJ, Dong BF, Xu LY and Li EM: Expression of fascin in thyroid neoplasms: A novel diagnostic marker. J Cancer Res Clin Oncol 134: 947-951, 2008.

14. Kostopoulou E, Daponte A, Terzis A, Nakou M, Chiotoglou I, Theodosiou D, Chatzichristodoulou C, Messinis IE and Koukoulis G: Fascin in ovarian epithelial tumors. Histol Histopathol 23: 935-944, 2008.

15. Iguchi T, Aishima S, Umeda K, Sanefuji K, Fujita N, Sugimachi K, Gion T, Taketomi A, Maehara Y and Tsuneyoshi M: Fascin expression in progression and prognosis of hepatocellular carcinoma. J Surg Oncol 100: 575-579, 2009.

16. Kostopoulou E, Daponte A, Terzis A, Nakou M, Chiotoglou I, Theodosiou D, Chatzichristodoulou C, Messinis IE and Koukoulis G: Fascin in ovarian epithelial tumors. Histol Histopathol 23: 935-944, 2008.

17. Gao X and Wu DH: Expression of FSCN1 protein in human epithelial tumor and its clinical significance. Nan Fang Yi Ke Da Xue Xue Bao 28: 953-955, 2008 (In Chinese).

18. Yamashiro S, Yamakita Y, Ono S and Matsumura F: Fascin, an actin-bundling protein, induces membrane protrusions and increases cell motility of epithelial cells. Mol Biol Cell 9: 993-1006, 1998. 
19. Xing P, Li JG, Jin F, Zhao TT, Liu Q, Dong HT and Wei XL: Fascin, an actin-bundling protein, promotes breast cancer progression in vitro. Cell Biochem Funct 29: 303-310, 2011.

20. Vignjevic D, Schoumacher M, Gavert N, Janssen KP, Jih G, Laé M, Louvard D, Ben-Ze'ev A and Robine S: Fascin, a novel target of beta-catenin-TCF signaling, is expressed at the invasive front of human colon cancer. Cancer Res 67: 6844-6853, 2007.

21. Hayashi Y, Toda K, Saibara T, Okamoto S, Osanai M, Enzan H and Lee GH: Expression of fascin-1, an actin-bundling protein, in migrating hepatoblasts during rat liver development. Cell Tissue Res 334: 219-226, 2008.

22. Pang QH, Dai XL, Wu WX and Zhang HX: Expression of fascin and CXCR4 in colorectal carcinoma tissues and their clinical significance. Chin J Cancer Prev Treat 18: 1175-1185, 2011.

23. Kong FL, Hu LX, Li CF, Huang MQ, Jiang YJ, Liao HW, Wu Y and Wang FX: The expression of fascin-1 in colorectal cancer and its clinical significance. Chongqing Yixue 45: 1519-1521, 2016.

24. Li C, Cai L, Zhang N and Zhang RQ: Expression and significance of fascin-1 in colorectal adenoma and colorectal carcinoma. Health Magazine 9: 11-12, 2015.

25. Liu L and Ding YQ: Expression of fascin-1 in human colorectal cancer and its significance. J Fourth MiI Med Univ 28: 108-110, 2007.

26. Ding Y, Liu L, Jiang HY and Ding YQ: Expression and significance of Tiam1, fascin-1, and HSPB1 in human colorectal cancer. Guangdong Med J 29: 230-232, 2008.

27. Li HW, Wang TS, Huang Y, Li YH and Hu HX: Expression of fascin-1 protein in colorectal adenocarcinoma and relation with clinicopathologic characteristics. Chin J Bas Clin Gen Surg 17: 362-364, 2010

28. Xue LY, Zou SM, Zheng S, Xie YQ, Wen F, Liu XY, Lin DM and Lü N: Expression of fascin and CK14 in different histological types of cancer and its differential diagnostic significance. Zhonghua Zhong Liu Za Zhi 32: 838-844, 2010 (In Chinese).

29. Chan C, Jankova L, Fung CL, Clarke C, Robertson G Chapuis PH, Bokey L, Lin BP, Dent OF and Clarke S: Fascin expression predicts survival after potentially curative resection of node-positive colon cancer. Am J Surg Pathol 34: 656-666, 2010.

30. Yao DY, Zhang HY and Xu X: Expression of fascin in colorectal carcinoma and its correlation with prognosis. Hainan Med J 25: 3089-3091, 2014.

31. Zhao ZY: Expression and significance of fascin and iNOS in colorectal adenoma carcinogenesis. Medicine People 7: 66, 2014.

32. Song XX: Expression and significance of fascin in canceration of colorectal adenoma. Heibei Med J 32: 2370-2372, 2010.

33. Yang Q, Lu ZH, Luo YF, Wan JW, Cao JL, Gao J, Wu SF and Chen J: Significance of fascin expression in colorectal adenoma and carcinoma. Chin J Diagn Pathol 13: 359-361, 2006

34. Yi LJ: Expression and significance of fascin and P27 in colorectal carcinoma. Luzhou Medical College, 2013.

35. Oh SY, Kim YB, Suh KW, Paek OJ and Moon HY: Prognostic impact of fascin-1 expression is more significant in advanced colorectal cancer. J Surg Res 172: 102-108, 2012.

36. Jung EJ,Lee JH, Min BW, Kim YS and Choi JS: Clinicopathologic significance of fascin, extracellular matrix metalloproteinase inducer, and ezrin expressions in colorectal adenocarcinoma. Indian J Pathol Microbiol 54: 32-36, 2011.

37. Duh FM, Latif F, Weng Y, Geil L, Modi W, Stackhouse T, Matsumura F, Duan DR, Linehan WM, Lerman MI, et al: cDNA cloning and expression of the human homolog of the sea urchin fascin and Drosophila singed genes which encodes an actin-bundling protein. DNA Cell Biol 13: 821-827, 1994.

38. Tubb BE, Bardien-Kruger S, Kashork CD, Shaffer LG, Ramagli LS, Xu JP, Siciliano MJ and Bryan J: Characterization of human retinal fascin gene (FSCN2) at 17q25: Close physical linkage of fascin and cytoplasmic actin genes. Genomics 65: 146-156, 2000.

39. Tubb B, Mulholland DJ, Vogl W, Lan ZJ, Niederberger C, Cooney A and Bryan J: Testis fascin (FSCN3): A novel paralog of the actin-bundling protein fascin expressed specifically in the elongate spermatid head. Exp Cell Res 275: 92-109, 2002.

40. Jawhari AU, Buda A, Jenkins M, Shehzad K, Sarraf C, Noda M, Farthing MJ, Pignatelli M and Adams JC: Fascin, an actin-bundling protein, modulates colonic epithelial cell invasiveness and differentiation in vitro. Am J Pathol 162: 69-80, 2003
41. Mosialos G, Birkenbach M, Ayehunie S, Matsumura F, Pinkus GS, Kieff E and Langhoff E: Circulating human dendritic cells differentially express high levels of a 55-kd actin-bundling protein. Am J Pathol 148: 593-600, 1996.

42. Grothey A, Hashizume R, Sahin AA and Mccrea PD: Fascin, an actin-bundling protein associated with cell motility, is upregulated in hormone receptor negative breast cancer. Br J Cancer 83: 870-873, 2000

43. Roma AA and Prayson RA: Fascin expression in 90 patients with glioblastoma multiforme. Ann Diagn Pathol 9: 307-311, 2005.

44. Xie JJ, Xu LY, Zhang HH, Cai WJ, Mai RQ, Xie YM, Yang ZM, Niu YD, Shen ZY and Li EM: Role of fascin in the proliferation and invasiveness of esophageal carcinoma cells. Biochem Biophys Res Commun 337: 355-362, 2005.

45. Hashimoto Y, Shimada Y, Kawamura J, Yamasaki S and Imamura M: The prognostic relevance of fascin expression in human gastric carcinoma. Oncology 67: 262-270, 2004.

46. Pelosi G, Pastorino U, Pasini F, Maissoneuve P, Fraggetta F, Iannucci A, Sonzogni A, De Manzoni G, Terzi A, Durante E, et al: Independent prognostic value of fascin immunoreactivity in stage I nonsmall cell lung cancer. Br J Cancer 88: 537-547, 2003.

47. Tong GX, Yee H, Chiriboga L, Hernandez O and Waisman J: Fascin-1 expression in papillary and invasive urothelial carcinomas of the urinary bladder. Hum Pathol 36: 741-746, 2005.

48. Fan G, Kotylo P, Neiman RS and Braziei RM: Comparison of fascin expression in anaplastic large cell lymphoma and Hodgkin disease. Am J Clin Pathol 119: 199-204, 2003.

49. Hashimoto Y, Skacel $M$ and Adams JC: Roles of fascin in human carcinoma motility and signaling: Prospects for a novel biomarker? Int J Biochem Cell Biol 37: 1787-1804, 2005.

50. Adams JC: Fascin protrusions in cell interactions. Trends Cardiovasc Med 14: 221-226, 2004.

51. Hashimoto Y, Ito T, Inoue H, Okumura T, Tanaka E, Tsunoda S, Higashiyama M, Watanabe G, Imamura M and Shimada Y: Prognostic significance of fascin overexpression in human esophageal squamous cell carcinoma. Clin Cancer Res 11: 2597-2605, 2005.

52. Kabukcuoglu S, Onedr U, Ozalp SS, Dundar E, Yalcin OT and Colak E: Prognostic significance of fascin expression in endometrioid carcinoma. Eur J Gynaecol Oncol 27: 481-486, 2006.

53. Xue LY, Hu N, Song YM, Zou SM, Shou JZ, Qian LX, Ren LQ, Lin DM, Tong T, He ZG, et al: Tissue microarray analysis reveals a tight correlation between protein expression pattern and progression of esophageal squamous cell carcinoma. BMC Cancer 6: 296, 2006.

54. Fu H, Hu Z, Wen J, Wang K and Liu Y: TGF-beta promotes invasion and metastasis of gastric cancer cells by increasing fascin1 expression via ERK and JNK signal pathways. Acta Biochim Biophys Sin (Shanghai) 41: 648-656, 2009.

55. Lu XF, Li EM, Du ZP, Xie JJ, Guo ZY, Gao SY, Liao LD, Shen ZY, Xie D and Xu LY: Specificity protein 1 regulates fascin expression in esophageal squamous cell carcinoma as the result of the epidermal growth factor/extracellular signal-regulated kinase signaling pathway activation. Cell Mol Life Sci 67: 3313-3329, 2010.

56. Li A, Dawson JC, Forero-Vargas M, Spence HJ, Yu X, König I, Anderson $\mathrm{K}$ and Machesky LM: The actin-bundling protein fascin stabilizes actin in invadopodia and potentiates protrusive invasion. Curr Biol 20: 339-345, 2010

57. Van Audenhove I, Boucherie C, Pieters L, Zwaenepoel O, Vanloo B, Martens E, Verbrugge C, Hassanzadeh-Ghassabeh G, Vandekerckhove J, Cornelissen M, et al: Stratifying fascin and cortactin function in invadopodium formation using inhibitory nanobodies and targeted subcellular delocalization. FASEB J 28: 1805-1818, 2014.

58. Li A, Morton JP, Ma Y, Karim SA, Zhou Y,Faller WJ, Woodham EF, Morris HT, Stevenson RP, Juin A, et al: Fascin is regulated by slug, promotes progression of pancreatic cancer in mice, and is associated with patient outcomes. Gastroenterology 146: 1386-1396.e1-e17, 2014.

59. Jass JR: Classification of colorectal cancer based on correlation of clinical, morphological and molecular features. Histopathology 50: 113-130, 2007.

60. Zhang JS, Li YC and Qi F: Expression of Twist and Fascin mRNA in colorectal carcinoma tissue. Shandong Med J 27: 42-43, 2015 (In Chinese).

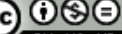

This work is licensed under a Creative Commons Attribution-NonCommercial-NoDerivatives 4.0 International (CC BY-NC-ND 4.0) License. 